\title{
SPECIAL ARTICLE Insights into the impact of sections and departments on your career: The APS-SPR Virtual Chat Series
}

\author{
Stephanie D. Davis ${ }^{1 凶}$, Jeffrey R. Fineman ${ }^{2}$ and Joseph W. St. Geme III \\ (c) The Author(s), under exclusive licence to the International Pediatric Research Foundation, Inc 2021
}

Pediatric Research (2022) 92:662-670; https://doi.org/10.1038/s41390-021-01825-8

Steve Abman (SA): We are pleased to welcome you to this session of the Joint American Pediatric Society (APS) - Society for Pediatric Research (SPR) Virtual Chat Series on pediatric academic medicine. The purpose of these "virtual chats" is to provide a forum that brings together diverse members of our pediatric academic community; these members represent different career stages, ranging from students, residents, fellows, faculty, and senior leadership. These "virtual chats" address critical challenges facing academic medicine. Previous sessions have included discussions about navigating career transitions, disparities in health care and outcomes, the dean's view of academic medicine, valuing and achieving diversity in academic medicine and other topics.

I am especially excited for today's session on "Insights into the impact of sections and departments on your career," which will address the vital impact of Section Heads and Department Chairs on one's career as these leaders balance achieving academic goals with meeting institutional demands. Their leadership positions are vital determinants of institutional success, but they also play central roles in the success of each member of their section and department. Thus, it is especially important to understand their goals for their sections and departments, expectations of their faculty, and to develop insights into how to best navigate one's career through opportunities provided by your Section Head and Department Chair.

We are pleased to have three outstanding leaders for today's panel. First, I would like to present Dr. Stephanie Duggins Davis, Professor and Chair of Pediatrics at the University of North Carolina at Chapel Hill, Physician-in-Chief for UNC Children's and President of the Society for Pediatric Research. Dr. Davis has had a wonderful career in research, clinical care, and administration through leadership positions in many academic societies, including Chair-elect for the American Board of Pediatrics (ABP) Board of Directors. She continues to be one of the major leaders in the field of Pediatric Pulmonary Medicine and has contributed to many NIH workshops and national professional societies.

We are also joined by Dr. Jeffrey Fineman, Professor of Pediatrics and Chief of Pediatric Critical Care at the University of California San Francisco. After completing his medical school and pediatric residency in New York, he moved to San Francisco for his fellowship in Pediatric Critical Care Medicine, which included research training at the Cardiovascular Research Institute (CVRI). As faculty, he has developed an international reputation as a leading investigator in pulmonary circulation and critical care cardiology, and is a major leader of research, education and training in national societies. Most importantly, Dr. Fineman has been recognized as an outstanding mentor and received the Maureen Andrew's Award for Mentorship from the SPR. In addition to developing an outstanding Section of Pediatric Critical Care with many successful faculty, Dr. Fineman also serves as the Vice Chair of Inpatient Services for his Department.

Finally, Dr. Joseph St. Geme, III is Professor of Pediatrics and Microbiology and the Chair of the Department of Pediatrics at the University of Pennsylvania, and Physician-in-Chief of the Children's Hospital of Philadelphia. Having trained at Stanford as an undergraduate, he completed medical school at Harvard, Pediatrics Residency at CHOP, and then returned to Stanford for his Fellowship in Pediatric Infectious Diseases. His extensive contributions to research, as well as training and mentorship, were recognized early in his career, and he rapidly rose through the ranks to become Director of Infectious Diseases at Washington University in St. Louis, Chair of Pediatrics at Duke, and then Chair of Pediatrics at CHOP. Throughout his career, Dr. St. Geme has been well-recognized as an outstanding investigator, educator and mentor. He has played major roles in many pediatric societies, including the APS, SPR, ABP, and the American Academy of Pediatrics. In recognition of his remarkable contributions to child health, Dr. St. Geme was recently selected by the Federation of Pediatric Organizations for the prestigious 2021 Dr. Joseph St. Geme Jr. Leadership Award, named after his father.

For today's seminar, we will begin with brief presentations from each of our panelists, before opening the question and answer session, starting first with Dr. St Geme.

Dr. Joseph St. Geme (JSG). I really appreciate the invitation to participate in this APS \& SPR chat. In considering the topic for this session, I asked the question, what role do Departments play in supporting the careers of faculty and trainees? And when I consider this question, I think back to my experience with the Chair of the Department at CHOP when I was a resident and just beginning to plot my career path, and then with the Chair of the Department at Washington University in St. Louis when I was a new faculty member and had to make some key decisions about how to prioritize my time and how to achieve success.

As a resident, I had the good fortune to work with Dr. Dick Johnston as Chair of the Department, now an Emeritus Professor at Colorado. He helped guide me into what turned out to be a formative fellowship experience in a basic science laboratory at

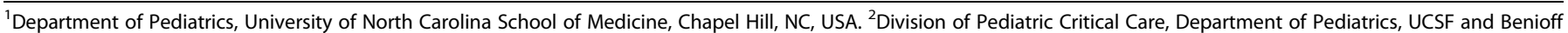

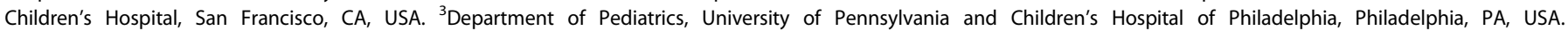
email: stephanie_davis@med.unc.edu

Received: 23 March 2021 Revised: 12 June 2021 Accepted: 10 August 2021

Published online: 25 November 2021 
Stanford, where I worked with Stanley Falkow, an incredible scientist and mentor. Dr. Falkow taught me how to be an investigator and in turn served as a mentor for me throughout the rest of my career, up until his death just over 2 years ago.

As a new faculty member at Washington University, my chair was Dr. Harvey Colten, an outstanding physician scientist, who showed particular interest in my success, or at least it felt that way to me, and provided invaluable guidance regarding protected time, decisions about specific grant applications, and achieving a balance between research and my interests in taking care of patients and teaching. Early on, he also invited me to join a junior faculty advisory committee, a group that included four or five other young faculty. We met with him every other month or so to discuss Department-wide issues. This advisory committee provided us, as junior faculty, with knowledge and insights that were otherwise reserved for Division Chiefs, allowing us to mature a bit faster than we might have otherwise.

As a Department Chair for the past 15 years (it's hard to believe it's been that long!), I think a lot about the role of the Department. In my mind, a fundamental responsibility for Departments is to foster outstanding patient care, to inspire education of the next generation of pediatric providers, to promote creative and important research that advances pediatrics and results in improved child health, and to advocate for children since children are unable to advocate for themselves. As I think about how to facilitate achievement of these priorities in patient care, education, research, and advocacy, the key vehicle is faculty and the key objective is to support the career development and the overall success of faculty.

What's necessary for the career development and the success of faculty members? Increasingly, it's important to consider faculty well-being, as well-being is a critical prerequisite for success. Accordingly, I think it's important to provide an infrastructure across the Department for well-being, a greater challenge than ever in the midst of the COVID-19 pandemic. In my mind, wellbeing initiatives need to consider both the workload/work environment and personal resilience. In terms of workload, the electronic medical record and regulatory requirements are significant challenges that we need to tackle broadly. At CHOP, we've been able to establish a Department-wide well-being initiative, with each Division having a physician lead for well-being to help guide local efforts. In addition, we've had some success in establishing hospital-wide efforts, with participation from all the academic departments and with support from the hospital.

Beyond well-being, it's important to think generally about the promotion process and facilitating efforts to ensure advancement of faculty. Along these lines, it's important to ensure that faculty understand expectations for promotion and receive the necessary guidance to achieve those expectations, recognizing that most institutions have multiple academic tracks and that each track typically has different expectations. Ideally, the Chair of the Department and the Committee on Appointments and Promotions (or whatever the local title of that committee might be) serve as important resources for individual faculty and also for Division Chiefs/Section Heads who are advising faculty.

In order to ensure an understanding of expectations for promotion and to ensure appropriate achievements, it's important to ensure mentoring, advising, and sponsorship within the Department. Departments can create mentoring and advising programs. At $\mathrm{CHOP}$, as an example, our mentoring committees are Division-based, but often include members from other Divisions, other Departments, and in some cases other schools at the University of Pennsylvania. We have a complementary advising program that involves pairing a senior faculty member with each junior faulty member, creating groups with a single senior faculty member and generally four or five junior faculty members. In all cases the senior faculty member is in another Division and hence in a position to provide unfettered, unbiased advice.
The Department Chair and the Division Chiefs can play a role as sponsors, nominating faculty for awards to promote their careers, creating opportunities for faculty, and connecting faculty with potential collaborators, as a way to advance their reputation and advance their career.

For faculty who have a research focus, the Department can provide grant review programs that will facilitate a rigorous and constructive internal review of grant applications before they are submitted for what is generally a less friendly, extramural review.

Similarly, the Department can provide research administrative and scientific infrastructure for faculty. Examples of administrative infrastructure include grant managers and examples of scientific infrastructure include research cores, key equipment, access to biostatistical support, and research seminars. These resources are invaluable and allow investigators to focus on what they do best as scientists, rather than spending time on administrative tasks that others can complete.

Departments can also provide workshops and other gatherings that support faculty development broadly. Some examples include teaching workshops, leadership development programs, education days, quality improvement report outs, and research retreats.

In addition, Departments can serve to promote a sense of community and can provide opportunities for social connections, whether in the academic setting, such as grand rounds, clinical conferences, research conferences, and department retreats, or through informal settings, such as luncheons, dinners, and other gatherings inside or outside the hospital. I think a sense of community is extremely important for almost all faculty, serving as a source of satisfaction and resilience. I suspect we've all struggled to maintain that same sense of community and the same network of social connections during the COVID-19 pandemic.

Beyond the role that Departments play for faculty and promoting faculty career development and faculty success, Departments promote the success of Divisions/Sections. One of my greatest joys as a Department Chair is to work with Division Chiefs to address faculty needs and to facilitate program development.

I'll conclude by saying that another way to think about how Departments support faculty and support Divisions is to consider some of the Associate Chair roles that exist in many of our Departments, or to also consider the range of cross-cutting functions within a Department. For example, in the Department of Pediatrics at $\mathrm{CHOP}$, as just one example, we have an Associate Chair for Clinical Affairs, Associate Chair for Education, Associate Chair for Research, Associate Chair for Patient Safety and Quality, Associate Chair for Information Technology, Associate Chair for Diversity and Equity, Associate Chair for Academic Affairs, and Associate Chair for Faculty Development. These are core aspects of the Department that represent areas where Departments can assist faculty in realizing their potential and achieve success.

(SA) Thank you for a great overview and perspective on academic medicine as a Department Chair. The first question is what attracted you to choosing to take the path to become an administrative leader, as a Department Chair, as opposed to perhaps other paths that you could have taken? What do you enjoy the most as Chair?

(JSG) I suppose it's fair to say that I've always enjoyed leadership experiences from the time I was young. I think there are a variety of aspects of being a Chair that were attractive to me when I first considered this role. I was fortunate to have a father who was a Chair, so I grew up understanding the joys of having this kind of leadership position, although certainly from a distance. More specifically, the opportunity to facilitate career development at a very broad level, broader than what I was able to achieve as a Division Chief, the opportunity to influence program development, and the opportunity to influence the field of pediatrics were very appealing to me and remain sources of great enjoyment. 


\section{(SA) Any major surprises?}

(JSG) Well, certainly as I learned more about the Department at Duke and then the Department at CHOP, when I first moved into these positions, there were some surprises and there were challenges that came up that I was far from prepared to address. I think most surprising and most challenging were some of the human resource challenges that exist within a Department, some of the tough decisions that relate to personnel.

(SA) Thank you. We will now hear from Dr. Davis.

Stephanie Davis (SD) Steve specifically asked me to identify trigger points that led me to choose a career, both as a Division Chief and then subsequently as a Department Chair. I'm going to briefly speak about these trigger points, and then focus on what Sections or Departments can do for you.

First, I never planned to become a Chief of a Pediatric Pulmonary Division or a Department Chair. My plan from an early age was to take care of children as a general pediatrician. Plans often change; the road is not straight, but often curvy. After completing a Pediatric Pulmonary fellowship at Riley Children's Hospital, I joined the faculty at UNC in 1999, and I really grew up as a faculty member in the Division of Pediatric Pulmonology at UNC.

It was the leadership and collegiality that were exceptional within this Division, highlighting what a Division or Section can do for you as a faculty member. The Division Chief, at the time I was recruited was Margaret Leigh and then Terry Noah. Both Chiefs were just incredible advocates for my career. This supportive environment ultimately pushed me and others beyond our comfort zone and I'm truly grateful for the mentorship and guidance that I received during these early years from leaders in pediatric pulmonary. These mentors pushed me to achieve goals that I never envisioned.

Early in my career, I realized that I really enjoyed building teams, conducting multi-center research, and being involved in national committees. In 2009, I was asked to be the Chief of Pulmonary at UNC, and then in 2011, I was asked to lead a larger Section of Pediatric Pulmonary Allergy and Sleep Medicine at Riley Children's, where I had completed my residency and fellowship.

I decided to accept these opportunities because I believed that as a Chief, I could serve as a mentor to others and help lead the next chapter for these strong Divisions, ultimately promoting respiratory health for children, both in North Carolina and in Indiana. In Indiana, I served as a Chief for 7 years, I had the pleasure of leading an incredible group of physicians through culture change, and we ultimately transitioned a very strong clinical section into a more research focused section.

As a Chief, I would ask each Division member what they wanted to do, why, and how I could help them achieve their career goals. I believe that one of the important impacts of a Division on a faculty's career is helping the faculty member delineate goals and identify passions. Serving as a Chief for a total of 9 years was a true honor. I think at Riley, we were also really able to make changes, due to an exceptionally supportive Department Chair; this highlights the importance of a Chair.

I have had exceptional mentors who really have served as incredible resources. One example is Wade Clapp, who is the Chair at Riley. He asked me very early in my tenure as Chief, if I was interested in being a Chair, and honestly, this was not a position I had really considered pursuing. Mentors, like Wade Clapp, encouraged me to pursue these leadership roles. I also participated in ELAM in 2016, and it was life-changing for me. This program really pushes you to define your goals. ELAM gave me the confidence to pursue a Chair position.

So why did I pursue a Chair position? My goal was to promote child health, and as a Chair, I would be in a better position to achieve this goal. It was never about the title, it was all about the goal, promoting child health and helping children. I accepted the position at UNC because I really wanted to make a difference in child health in my home state of North Carolina. So, when asked, what can a Section or Department do for you? Let me highlight six themes.

First defining the vision and mission for the Division or Department is critical. This often occurs through strategic planning groups; these groups can really represent the faculty's interest and guide development of the mission and vision. You can then use the vision and mission as your North star. By focusing on this North star, decisions, no matter how difficult, really become very clear. The vision and mission of the Division or Department certainly impact the future. However, a strong Section and Department should have resources to help individuals achieve their personal mission and vision. This may be facilitated through faculty development or mentoring groups. A strong leader acts as a connector for faculty members and chiefs. Connecting people may be helpful because these relationships empower others to achieve more, which is especially true in the era of team science and multi-disciplinary care.

The second theme is mentorship and sponsorship. Advocates can help you see abilities in yourself that you may not be able to identify. Frankly, this theme of mentorship and sponsorship has been highlighted throughout our virtual chat series. A strong Division or Department creates an environment valuing research, clinical care, education, and advocacy. Mentors, sponsors, Chiefs, and Chairs can help provide resources for faculty members to develop their goals. For the young physician scientist, this may mean providing protected time through internal funding or foundational funding until he or she is awarded independent funding.

A third theme is to focus on specific objectives. Make sure you do not have too many objectives. Progress can be impaired if you have too many objectives. You want to outline these expectations or objectives with individual faculty members or Chiefs.

A fourth theme is to listen, listen, listen, and achieve consensus. Often as a leader you really need to actively listen to fully understand the culture of the Division or Department. There is a book written by Jeff Houpt and Susan Ehringhaus entitled Learning to Lead in the Academic Medical Center: A Practical Guide that really highlights the importance of culture! Active listening is also really important to ensure that faculty members have a voice.

A fifth theme is to recognize both small and big achievements. A healthy Department or Section value their faculty; highlighting the value of faculty members often does not occur through financial incentives. It is important to display gratitude to those that lead all missions, clinical research, education and advocacy. Highlighting this work is important. You can do this verbally at meetings, through newsletters, or through handwritten notes. A strong Section or Department also empowers others to shine. It is not about you as a leader. You really are there to empower others to lead.

Finally, the sixth theme is to support and promote work-life integration. I am married to an ENT surgeon. I have three wonderful sons. I have certainly experienced the difficulty of balancing personal and professional life. I personally could not have served in any of these leadership roles without the support of colleagues who stepped in when I needed to go to my son's baseball game or attend school events. I truly believe the best resource of any Division or Department is its people.

I will leave you with one quote from Eleanor Roosevelt, who said, "you must do the things you think you can't do." I really think Sections and Departments can empower faculty to push beyond their comfort zone, ultimately achieving their academic goals.

(SA) Thank you, Stephanie, for your insightful thoughts. First, I would like to ask you how important was the ELAM program for your career and can you explain what opportunities the ELAM program provided? 
(SD) Absolutely, ELAM, an Executive Leadership Program in Academic Medicine, through Drexel University, is specifically for women who are interested in academic executive leadership roles. It is a phenomenal program. Approximately 50 women participate annually. It is a yearlong, intense program where you gain leadership skills, define your goals and push yourself to consider institutional leadership roles. ELAM really helped me personally define my ultimate career goal. ELAM is also helpful because you network with many women leaders across the country. This type of networking is very beneficial. It is a competitive program and typically each institution will only send 1 or 2 women if they are accepted into the ELAM program.

(SA) You described your career decisions and choices that shaped your career, and we have many people in the audience who are likely beginning to think about the possibility of becoming a Department Chair. If you are being recruited for any faculty position, but especially if you are thinking about perhaps becoming a Chair, what should you consider most? Also, how does one prepare to take the path that you did?

(SD) As a faculty member, when you are looking at positions, important characteristics to consider are mentorship and sponsorship. How strong is the mentorship or sponsorship at the institution? What is the culture of the Division or the Department that you are joining? Culture is specific to the Division or the Department. For example, we like to refer to the Carolina Culture at UNC. Are the leaders accessible and supportive of faculty members? Does the Division or Department have clinical, educational, advocacy or research programs that really align with your goals? Are the faculty engaged? Are they happy? Do they feel that their voices are heard? Are goals and objectives well defined to move the Division or Department into the next decade? What is the vision of the Department or Division, and what is the relationship of the Division or Department with the hospital or health-care system? It is important to understand the institutional structure, and the relationship of the medical school with the health-care system.

If you are considering leadership roles, does the institution promote leadership programs? I would consider attending leadership programs through the AAMC, ELAM, or Harvard. Are there mentors or sponsors, who can help connect you to others in your field of interest? I think these are just some of the issues to consider. There are many others, but due to our time limitations, I am unable to go into any more detail.

(SA) Thank you so much. We will now hear from Dr. Jeff Fineman, who has been spectacularly successful as a Section Head in developing an outstanding section with successful academic faculty members. We are delighted to hear from Jeff.

(JF) Thank you, Steve for those kind words. I am humbled to be part of this group. I do not thank you for having me go last. This is like having to follow the Rolling Stones! One sure thing that I learned so far is that if you want to be a Section or Division Chief that supports the academic mission, all you have to do is try to get a job with Stephanie or Joe, and I think you will be all set. All kidding aside, in order to be a successful section chief, you have to be on the same page with the Department Chair, and she or he has to be supportive of what you want to accomplish.

I thought I would provide you with a little introduction as to how I became involved with the academic mission and subsequently mentoring. I was one of those folks that went straight through, from undergraduate studies to medical school to a clinically heavy pediatric residency at Bellevue in New York, followed by a chief residency year. So, when I moved to join UCSF and the Cardiovascular Research Institute for fellowship training, I had absolutely no research experience and did not know anything about academic medicine. When I arrived, I was lucky enough to be introduced to Dr. Rudolph, who gave me the opportunity to work within this incredible infrastructure that he had built to conduct large animal physiology studies and investigate the pulmonary circulation. I was extremely lucky. I was studying a substance that was a pulmonary vasodilator that turned out to work via endogenous activation of something Steve and I would call ERDF initially. ERDF turned out to be nitric oxide.

Warren Zapol at Massachusetts General then called me and told me that they were going to start a clinical trial on the use of inhaled nitric oxide for newborns with persistent pulmonary hypertension and asked if I would participate. Sam Hawgood was the head of Neonatology at the time. I was straight out of Fellowship and this young ICU guy walks into the NICU and asks Dr. Hawgood if we could enroll neonates and participate in this study. At the time, Dr. Hawgood was working with Dr. John Clemons, who had a lot to do with the discovery of surfactant. In those days, they were doing a lot of surfactant clinical trials, and they were in the middle of a surfactant clinical trial at the time. When I spoke with Dr. Hawgood, he said that "although the ongoing surfactant trial overlaps, this seems like a really innovative and important trial, so we're going to put our surfactant trial on hold, and let you do this trial. Plus, it will be good for your young career". It was that kind of collaboration, mentoring, and support that I have never forgotten. Dr. Hawgood ultimately became my Chair, and then my Dean, and now our Chancellor. It was this unselfish, passionate, and supportive environment of an academic and physician-scientist model that I was so lucky to be exposed to early in my career. I have just tried to carry that forward as much as possible.

With that as background, I thought we could move into the weeds a bit as to what I think about the role of the Division Chief in supporting academic medicine. First, let me state that what I am going to say is really nuanced depending on the structure of your system, including the divisional structure, departmental structure, and their relationship with the hospital. With that disclaimer, there are several competing factors that you have to try to balance. One is trying to find the balance between supporting the academic mission and balancing the Divisional financial books. I don't think you can go into any Chair's office or certainly any departmental business person's office and try to make the argument that supporting the academic mission by hiring several people that have a lot of academic promise, but don't participate in clinical work, is going to pay off financially. That is a tough sell for sure. You could say that you think they are going to ultimately be a R01 funded investigator, but that will only support $25 \%$ or $40 \%$ of their salary. If you hire a full-time clinical person, they can generate a lot more RVUs. So, it is a very difficult business argument to make. You need to determine the best approach to balance the Divisional books. There is no question that within critical care, hiring a smaller number of full-time clinical people is more cost-efficient.

The next challenge is to create an environment of equity within the Division, despite faculty participating in different roles. This includes faculty focused on excellent patient care, faculty focused on research, faculty focused on the educational mission, etc. These faculty may all be participating in different amounts of clinical time. How do you make sure that everyone appreciates the Division or the Section's different missions of great patient care, innovation and research, education, and advocacy? Faculty can be participating in very different activities, with equal importance as it relates to our mission. How does everyone understand and appreciate this? I think it is really important to spend a lot of time trying to build that culture and environment, where everyone understands the differing jobs, but acknowledges that everyone works hard, and collectively all these jobs benefit the mission.

Another challenge involves balancing the clinical needs of the Division. You obviously have to take care of the patients while protecting individual faculty's research time. In addition, how do you ensure clinical competence while protecting faculty's research time. So, how do you make sure, for example an ICU doctor participating in 6 or 8 weeks a year of inpatient work, is still strong 
666

enough clinically? Those are just some of the challenges that we face as Division/Section Chiefs.

I will try to review some of the solutions rather quickly. I personally think that we cannot make a financial argument in terms of balancing the books. We certainly must do everything to optimize our revenue; optimize RVU generation, billing, intra and extramural funding, etc. I think that it is also important to try to form partnerships with industry and philanthropy. I think it is very important that you protect your junior faculty upfront and make enough revenue to support them. I think the model of having junior faculty participate in a full clinical load upfront, and then having them "buy down time" as they acquire grant support is somewhat of a "catch 22." If they are doing a full clinical load, it is very hard for junior faculty to have the time to really embark on an academic career and generate the preliminary data needed to acquire funding.

Obviously, optimizing the chances of successfully acquiring career development awards is a big goal. So, identifying potential candidates early in residency and extending training periods within training grants, such as through T32s, particularly if they are just M.D.s, and not M.D./Ph.D.s is crucial. You want to provide protected time to the trainees and junior faculty so that they are adequately prepared to compete for $\mathrm{K}$ awards. This also includes K12 awards, whether it's a departmental K12, or other K12 awards that are available for these young scholars.

Importantly, as has been highlighted by both Joe and Stephanie, excellent mentorship is vital and having Departmental infrastructure that ensures adequate mentorship, both individually and at a departmental level is crucial. I think an approach that we have taken, that has been successful, is that we've used the entire UCSF faculty community, not just a Division or the Department for mentorship. Since we are not a freestanding Children's Hospital, we remain close with our adult colleagues and they have been a great source of mentorship. In addition, we utilize faculty mentorship from the campus at Berkeley and the affiliated research institutes, such as the Cardiovascular Research Institute and the Gladstone Institutes. So, utilizing the full breadth of the associated University, I think is important.

Creating an environment of transparency and equity is vital. In terms of equity, I think salary is very important. For our division, salary is based on academic rank regardless of your role; clinical, research, education, etc. So, it does not matter whether you are doing 6 or 8 weeks in the ICU or 16 weeks in the ICU, the pay scale is the same. Everyone is working hard, and everyone is really trying to accomplish our divisional mission. For our division, the ICU does in-house night call which we all see as a physical and emotional burden despite receiving bonus payments for night call. Therefore, this "burden of night call" is divided equally amongst the faculty; a research faculty member who is doing 6 clinical daytime weeks does the same number of nights as the fulltime clinical faculty member who is on service for 16 clinical daytime weeks.

Most importantly, I believe it is simply that everyone understands and appreciates the different roles each faculty member plays in our mission and the different nuances for each role. For example, when your focus is research, although you are doing less clinical time, I like to say that "your list is never done". There is always more data to analyze, something to write up, etc. You never go to bed without having more research activities to do. When your focus is clinical medicine, although you are doing more clinical time, which is difficult and less flexible, once you are done seeing patients and your notes are done, your list could be done for that day. So, there are advantages and disadvantages of everything that we choose to do, and everyone just has to appreciate and respect these differences. The clinical competence aspect can be tricky, but I believe we must continue to assess this issue and adjust as needed. For example, since we often train many faculty that we retain, you may identify a physician-scientist during fellowship training who is embarking on an academic career and you may want to have them do some more clinical time if you think that they need more clinical training, or even assign a faculty clinical mentor to help them through the transition to attending physician. Lastly, to reiterate, I think it is really important, for whichever system you have in place at the Divisional or Sectional level, that you do your best to make it "fair" and transparent. Obviously how people define "fair" may differ, but we try our best.

(SA) That was excellent Jeff. I was so impressed that you were "a late bloomer" in developing your research interests, and you emphasized the importance of mentorship and opportunity, especially within the Cardiovascular Research Institute. With the growing interest in team science and interdisciplinary training, I was wondering if you could briefly describe the Cardiovascular Research Institute and its remarkable success as an early model for this approach. We will then ask Stephanie and Joe to weigh in on the topic of team science and career development.

(JF) I think the real secret for the Cardiovascular Research Institute has been the focus on mentoring, and supporting the physician-scientist model, which has simply always been a priority; people just cared about this. The Cardiovascular Research Institute has always made team science a priority, and collaboration between M.D.s and Ph.D.s has always been encouraged and facilitated. I think that has been very important and certainly fundamental to my success. Therefore, I loved the move to the multi-PI format for $\mathrm{NIH} \mathrm{R01s.} \mathrm{I} \mathrm{think} \mathrm{this} \mathrm{was} \mathrm{quite} \mathrm{clever} \mathrm{and}$ facilitates a much more collaborative scientific environment and encourages team science.

(SA) Thank you. To continue the theme of team science, I would like to ask whether Stephanie and Joe can give us other examples of successes and challenges of team science. One issue is how should Department Chairs and Promotion Committees address individual career development beyond the traditional metrics of first or senior authorships, as many contributions may be vital to the success of a project, yet lead to only middle authorship?

(SD) I strongly agree with Jeff's comments about Multi-PIs. I, along with other faculty within the Department, have been a MPI on many grants, and the collaboration among the Pls makes a huge difference in both acquiring awards and facilitating research progress. You just simply achieve more together as a team. I will share an example of where team science made a difference. UNC sits in the middle of the Campus, so we can work with both M.D. and Ph.D. investigators across campus. A few years ago, we submitted an airway modeling grant to $\mathrm{NHLBI}$, and the team consisted of a physicist, a mathematician, a computer scientist, an ENT physician, and me. We were awarded a R01 to evaluate and model the upper airway. I learned a great deal from the investigators within this team. We were able to publish some great work through this team science approach. There are many other examples highlighting the advantages of team science.

(SA) Joe, you have done a wonderful job leading $\mathrm{CHOP}$, which has extensive programs in laboratory and translational research as well as success with developing team science. Perhaps you can comment on the challenges of team science within your department.

(JSG) Yes, I think there are challenges within the Department and challenges within the School of Medicine and the University regarding how to best recognize team science. The key is for Schools of Medicine and Universities to identify ways to credit individuals who have participated in team science, which sounds easier to execute than it actually is. Faculty need to be able to describe their contribution to a multi-author paper and to a multiinvestigator project stemming from team science. There is no doubt that team science is essential for research advances, bringing together complementary expertise and complementary perspectives in interdisciplinary work. The number of great 
examples of team science continues to grow, making the case for the importance of team science and for recognizing faculty participation in team science.

As an example of great team science, I would cite the work of our group at CHOP studying very early onset inflammatory bowel disease. This work involves gastroenterologists, who are on the frontline seeing these patients and are studying the microbiome. In addition, there are human immunologists and basic immunologists who are making important contributions in dissecting the relevant immune pathways. There are geneticists who have participated in the genome sequencing and the annotation of the genomes of individual patients. There are bioinformaticians who play a critical role in analyzing the vast data from the microbiome and from the genome. It is due to the involvement of all these individuals that this group has been able to make the advances that it has, alongside the work of other groups across the country. If it were just the gastroenterologists, we would not know what we know. If it were just the immunologists, we would not know what we know.

(SD) For promotion committees, just being focused on first and senior authorship is "old school" to me. We really must change promotion policies. I think this is happening across the country. Frankly, it takes a large team to conduct multi-center work and all contributors need to be recognized for their work.

(JSG) This is a role that Department Chairs and Division Chiefs can play, making sure that deans and school of medicine promotion committees understand the importance of multiple contributors to projects in order for advancement to take place.

(SA) More faculty now have marked interest in informatics and working with the electronic health records. Do you have specific approaches and advice for your faulty to help them develop successful academic careers for promotion in these areas? How would you encourage them to succeed in such non-traditional tracks, especially as such individuals may be vital for both the hospital and Department?

(JSG) Well, most schools have multiple academic tracks and expectations for each of these tracks are different. I think a key is to identify the right track for each individual faculty member, recognizing that sometimes there is not a perfect track. If a faculty member is on a track where the expectations are aligned with that individual's expertise and scope of work, it is much more straightforward for the individual to advance.

At the University of Pennsylvania, there is a tenure track and there is a clinical investigator track (called the "Clinician-Educator" track). The expectation for individuals on the clinical investigator track is that they will typically pursue collaborative investigation, maybe as a clinician, maybe as a clinical trialist, maybe as a biostatistician or as a bioinformatician. People on this track may be the principal investigator driving a clinical trial or may be providing other critical contributions as a co-investigator. People on this track may be first author, a middle author, or the final author on publications, in all cases receiving credit towards promotion based on their contributions.

(SA) Stephanie has done a remarkable job within her department empowering faculty who play vital roles in health care in the areas of advocacy and community engagement, especially in issues of equity, diversity, and inclusion. Are there specific approaches that you use to help develop careers that do not follow traditional academic paths yet are important functions for the department?

(SD) That is a great question and important. We have a Vice Chair for Diversity, Equity, and Inclusion, within our Department. We also have several committees throughout the school and the Department where people can lead in this area. To promote recruitment of diverse faculty, a member of the Department's Diversity, Equity and Inclusion Committee serve on all search committees. This is a critical area for all academic missions. We strive for diverse faculty; recruiting diverse faculty is a priority. In terms of promotions, how do you highlight this work? Certainly, leadership roles within these committees, serving as an author on position papers, or as part of commentaries are examples of academic productivity. It is important to highlight this work on your CV and delineate your role. For committees, you can describe your role as a member, leader, moderator, or presenter at national meetings. I also think, Steve, this highlights how we need to change our thought process about promotion. Promotion should not just focus on first and senior author papers. There are many other important roles that can be highlighted on the CV. You can also compete for advocacy grants within your state or participate in your state AAP chapter. Within our pediatric residency, we also provide opportunities to participate in diversity, equity and inclusion initiatives, as well as advocacy opportunities. It is important to educate our promotion committees as we think about these initiatives moving forward.

(SA) Each of you spoke about the importance of mentorship and sponsorship, especially for developing leadership opportunities for underrepresented minorities. Do you have examples of specific programs for promoting underrepresented minorities in medicine that provides successful sponsorship and coaching to enhance academic leadership?

(JF) This is clearly a vital issue. At UCSF, our Chancellor created an Office led by the Vice Chancellor for Diversity and Outreach, which is responsible for a great deal of innovative programs. One such initiative developed within the School of Medicine is the "Differences Matter Initiative." Within the Division, I am lucky to have several Diversity champions amongst the faculty, who run several programs. For example, every member of the division must undergo our 2-day diversity training program, and we have several "book clubs", "movie clubs", and didactic lectures focused on DEI issues. In addition, as a department, we divided into groups and participated in the 21 Day Racial Equity Habit-Building Challenge. We have several other divisional initiatives and have formed sub-groups to work on different aspects of advocacy. For example, I am on the research advocacy portion that focuses on attracting more underrepresented minorities into academic medicine. As you all know, this is work in progress.

(SA) Joe, can you comment further on how to best promote diversity within careers in academics?

(JSG) It is such a critical area, and it is important for us as leaders to establish diversity as a priority. I have to say, I feel very fortunate that we have an incredibly bright and energetic leader in the Department of Pediatrics at CHOP, Angie Ellison, who has been in her role as the Associate Chair for Diversity and Equity for roughly 3 years and has tremendous leadership ability. She has brought remarkable leadership and creativity to this area. She has advanced efforts across the Department and the hospital to promote diversity in the process of recruiting residents, fellows, and faculty. In addition, she has strengthened the pipeline, with a Medical Student Summer Scholars Program that provides first-year medical students with clinical and research experiences in the Department. She has also established an opportunity for faculty to apply for Departmental support for Division-based and Department-based projects that promote diversity and equity, whether as a quality improvement project, an education project, a clinical project, or a small research project. She has also highlighted that we need to provide protected time for faculty who assume significant responsibilities related to diversity, offsetting the minority tax. These are a few examples.

(SA) I would like to turn to the important issue of the "vanishing clinician-scientist," with which we all struggle. The importance of strong mentorship, team science, and collaborative environments have been discussed. Another question is how do you balance protected time early in a junior faculty's career with reduced clinical duties, yet this is also a critical window for developing foundational clinical skills and confidence as a physician?

(JF) The "vanishing clinician-scientist" is a very important issue. I believe that even at the most academically oriented medical 
centers, the academic mission is no longer "a given". There is so much discussion about clinical growth and RVU generation, which unfortunately academics is often put aside and requires an active process of discussion and support. I feel strongly that protecting time early in a faculty member's career is crucial to their success. Waiting for them to "buy down time" with grants is very difficult if they do not have the time upfront to generate data for their grants. There are certainly many ways to accomplish this financially; none particularly easy. Of course, utilizing philanthropic monies to support the academic mission is extremely helpful, but often difficult to obtain. Optimizing intramural funding opportunities is important, and affiliations with industry can also be helpful. Additionally, if the Department sets RVU targets, I argue that they are divisional goals and not individual goals. That allows more senior faculty to take on more of the clinical time to help support the junior faculty. Lastly, it would be great if the hospital appreciated the importance of academics not only as an important part of the mission, but a piece that attracts patients from other locations (i.e. outside your state) to the hospital. We must commit to just keep fighting the fight. Obviously, we need to optimize our sources of revenue to support the academics. I do not take that for granted.

(SA) So, can we explore further the balance between academic missions and the more corporate goals? You are all active academically and outstanding academic leaders. How do you approach this balance? Has there been a real switch in this balance? In other words, given that clinical dollars may fund the academic mission, how do you handle this issue at the institutional level, at the medical school level, with the Deans and other Department Chairs? Joe do you want to start?

(JSG) Yes, I think striking the balance between hospital financial performance and the academic mission is an issue nationally. I think it is important for all of us to emphasize that there's a relationship between scholarship, advances in biomedical research and high-quality clinical care, which ultimately attracts patient populations that generate revenue. So, in fact the academic mission and hospital financial performance go hand in hand, an important concept for Deans, for hospital CEOs, and for health-care system CEOs to recognize. There are many examples where research programs create visibility for the institution, for the department, and for the related clinical program, ultimately attracting patients. As we think about potential revenue streams for our hospitals and health-care systems, we need to emphasize the relationship between biomedical research and the potential for intellectual property, the potential for commercialization, and the potential for philanthropy.

(SD) In any health-care system meeting, the Departmental Chairs highlight the importance of research and the academic missions; that is your job as the Chair. Our health-care system is currently hiring a research leader for the entire system, which will help bridge the medical school and the hospital entities. I completely agree with Joe, it is the clinical trial work and other research initiatives at your academic institutions that attract patients and ultimately may lead to national visibility.

I also think philanthropy can help support young physician scientists when they are competing for their first $\mathrm{K}$ Award. So, as a Chair, along with the Chiefs, we work closely with our philanthropy group, because we understand the importance of philanthropy dollars in helping to provide protected time for these physician scientists.

(SA) Do you sometimes feel that faculty members, either in the Section or in the Department, are not fully aware of resources? When should faculty members reach out for help? I sometimes wonder if junior faculty are not sure when to ask or how to negotiate for help.

(JF) Our institutions are complicated, making it difficult for faculty, almost regardless of the level of experience, to know about all the resources that exist. I think the more communication that exists among faculty across divisions, across Departments, across schools, the better. I think more interaction with faculty and leaders, such as Section Chiefs and Chairs, who may have a broader knowledge, will lead to a better understanding of resources. I think there are lots of opportunities for faculty within different Divisions to collaborate because of overlapping interests. Critical care medicine and pulmonology, or pulmonology and neonatology as examples; there are lots of opportunities and these collaborations will be of benefit to every member who is in that collaborative community.

(SD) I completely agree with Joe about communication. I am often surprised. A faculty member may say, "I didn't even know about that grant opportunity." It is because we are overwhelmed with e-mails and meetings. I think that it is nearly impossible to overcommunicate since communication is extremely challenging within our institutions. So, you either communicate with your Chiefs as a Chair, communicate to faculty within your Pediatric Department meetings or communicate to faculty and staff throughout the entire Children's hospital. We communicate and communicate, but still, messages may get missed.

(JF) Yes, I could not agree more. Even at a Divisional level with just 32 faculty, I find myself needing to communicate with the faculty much more often than I previously did. I often find myself thinking: "Why didn't I forward the e-mail about this particular grant to this particular person, or I should have nominated this particular faculty for the SPR, for example". So, I just implemented a form that's pretty extensive for each faculty member to complete that contains career goals, updates, etc. Also, we just changed the frequency of our meetings from annual to every 6 months.

(SA) Jeff, you spoke about equity within a Section, in terms of those who may predominantly participate in clinical work compared to those who predominantly do research. How do you keep them from being split into different worlds and maintain a sense of collegiality and collaboration?

(JF) I have been very lucky in the sense that the culture within the Division has always been one of appreciation for the academic mission and the differing roles that each faculty member plays. To maintain that, we try to respect and appreciate everyone's role. For example, at each divisional faculty meeting we start by announcing any honors or awards. This is not limited to grants and publications, but importantly includes any clinical care acknowledgments and teaching awards. Lastly, we try to be as "fair" and transparent as possible.

(SA) One of the questions from the audience is regarding your thoughts about global health initiatives, which are not usually big money magnets for divisional funds. However, these activities are a substantial part of academics these days.

(SD) We have a large Global Health Center at UNC. I strongly support global health initiatives, and we, just appointed a Director of Children's Global Health. Because we have leaders and scientists across multiple Divisions and Departments that work within global health, we have not only interacted from a collaborative perspective across the University, but also, worked with philanthropy, because there are a number of different donors that really want to support children's global health.

(SA) Joe, can you comment on your approach for supporting academic careers in global health in your Department?

(JSG) Yes, I will first say that I agree with what Stephanie is saying, that global health is really a priority. It is a growing area of interest for students and residents. Consequently, in order to attract the best and the brightest into pediatrics and pediatric subspecialties, it is very helpful to have a global health program. In addition, all of us want to improve child health, and we can improve child health by taking care of patients who walk through the doors in our hospitals and our clinics. We can also improve child health by conducting research that has applicability to broader populations. We can improve child health by providing care in underserved areas across the globe and identify 
approaches to care that have broad applicability to low- and middle-income countries.

As Stephanie says, global health is an area that is attractive to some donors, resulting in philanthropy to support global health efforts and offset some or all of the expense.

We have used philanthropy to a significant extent to allow our global health program to grow.

In considering academic careers, there are a variety of available academic career paths in global health, ranging from a patient care focus to an education focus to a research focus. A common approach is to establish a relationship with an academic institution in a low- or middle-income country.

(SA) If you were mentoring someone who early in their career told you that they wanted to be an academic leader or a Department Chair, how would you advise them?

(SD) First of all, Steve, I really think it is not about the title, but it is about your goals and passions. So, for me, my goal was to promote child health and I thought, in a Chair role, I could achieve this. My advice would be to first identify strong mentorship and sponsorship. Mentors, sponsors, Chiefs, and Chairs may advocate for you and help you form connections with others, not only within the Department and Division, but nationally. I would advise to participate in leadership programs and define your career goals. From my perspective, it is about where you really want to make a difference. That may be as a physician scientist, or it could be through a clinician-educator route. There are a lot of different routes to achieve academic goals. I also think coaching through either internal resources or executive coaching, can be helpful. Finally, if you are interested in leadership roles, do not undersell yourself. I think too often, especially as pediatricians, we have a little bit of the imposter syndrome at times. So, having sponsors and mentors who push you can be helpful.

(SA) Thanks, Stephanie. Joe, do you have a comment?

(JSG) Yes, I would echo what Stephanie has said. In addition, I would say, achieve success in what you are doing. Be as successful as possible, whether as a clinician, a clinician educator, a clinician investigator, a laboratory investigator. Look for opportunities to assume a leadership role, maybe leading a clinical program or leading a training program as a first step. Aim to gain visibility nationally through your academic success. Finally, having a role model and a sponsor can be very helpful.

(SA) Since you work so closely with managing the business aspects of running a department, do you have advice on business courses, the value of getting a MBA, and how does that all fit into one's already busy academic life?

(JSG) Well, I have certainly wondered along the way if I should pursue a MBA.

I never have. I have leaned on very strong financial administrative leaders, and I have learned a lot from them, listening carefully, paying attention to details, and asking questions. I suspect I could be stronger in certain areas if I had a MBA, but it is a matter of making choices and finding time. I have elected to maintain my research program throughout my career, perhaps instead of pursuing a MBA. I have elected to continue to see patients and to engage in teaching as a Chair, like many others have, but, again, that is a choice that I made, instead of earning a MBA. So, I have focused on learning about business and finance through practical experience and through latching onto a very knowledgeable, experienced, and capable partner.

(SD) Similar to Joe, I have continued my research program instead of obtaining a MBA. I certainly learned quite a bit through some of the leadership courses I attended. I am not afraid to ask financial questions. I surround myself with people who are knowledgeable and who have a financial background. I agree with Joe, if I had pursued a MBA, it may have been super helpful. However, I have really focused on my research career, and the work that we are doing in early lung disease. I have also focused on building the physician scientist pipeline.
(SA) Great, very helpful, Stephanie. I am going to ask Jeff how he acquired his business savvy to run a Section, balance the books and understand RVUs. It was all from the streets of New York, I think, right?

(JF) I wish. I am not very business savvy at all. I must say, I should probably have gotten a MBA. As both Stephanie and Joe said, I tend to depend on people that I trust and are very good at finances. More recently I have had to pay more attention to the finances, and that is not something that I love to do, quite honestly.

(SA) We have covered many issues. I would now like to discuss topics related to human resources, and the special skill set that is needed to navigate differences in style and personality. How does one best manage conflicts? Could you share insights about conflict resolution and how to best approach such difficult situations? These may include troubled faculty, aging faculty, and many other issues.

(JSG) Sure, I think human resources and personnel issues, are among the most challenging for a leader. I would hardly profess that I am the best at this. I think I have learned a lot over the past $15-20$ years.

I think it is important to appreciate that variety is what makes the world go 'round' and that diversity is good. In some cases, it is just recognizing that a faculty member who may be an outlier in terms of his or her thinking, is making important contributions and adding a valuable perspective. I need to find a way to be more accepting of what that individual is contributing. I have found it very helpful to have great human resource partners and to get advice from colleagues who have high emotional intelligence and are good problem solvers.

I am not bashful about going to a human resources specialist or leaning on others within the Department who have great insights, maybe a Division Chief, maybe a senior administrator. I think it is often very helpful to get input from these individuals. I try not to solve these problems all by myself, but instead engage other people. That has been my own approach, but it can be a challenge.

(SD) I am not shy about calling our human resources office. I also have learned that there are always two sides to every story. I also ask faculty members who are struggling if there is something else going on. It has really been helpful, understanding the entire story, not just one side. Human resource issues are challenging. I often speak to other advisors within the Department, as well as within the human resources office to help navigate difficult issues. Unfortunately, you have to make really tough decisions. This is one of the hardest aspects of being a Chair. However, not making tough decisions, can lead to more difficulties. This is where human resource advisors can help you navigate the process.

(JF) I just could not agree more with the past two very thoughtful comments. Dealing with human resource issues have been the scariest and the hardest thing to do, and the area that I felt the least prepared. Fortunately, I am not the last word on these matters, so I get help from a lot of well-trained people. Since these issues are very difficult for me, I tend to avoid these difficult conversations, so I have to remind myself to be upfront, to be ahead of it, and not avoid these important issues. So that's just a personal reminder to myself, because I do find it very, very difficult.

(SA) Here is a final question from our audience: one of the threats to the physician-scientist pool is the loss of funded physician-scientists to administrative leadership roles. In other words, the most successful leaders in research, who best understand what is needed to succeed as a physician-scientist, can end up with extensive administrative responsibilities. Without that basic experience in research, how do hospital, departmental, and medical school leaders, make decisions to support academics?

(JSG) I think it is important for leaders in Pediatrics, Department Chairs, Division Chiefs, Associate Chairs, to continue to be investigators. It is hard work, but there are ways to do it. For me, pursuing research and participating in the process of 
discovery is extremely satisfying and adds a critical dimension to what I do each day, hopefully allowing me to be more effective in my administrative roles. As faculty move into leadership roles, we need to emphasize that they can maintain their research program. They may need to manage it a bit differently than in the past, devoting time to their research program at different times of the day or on different days of the week. We need people who are in leadership roles to continue to be active investigators and to be advocates for research in discussions with the hospital, health-care system, and School of Medicine leaders.

(SA) Once again, I wish to thank our panelists and audience for an outstanding session.

\section{COMPETING INTERESTS}

The authors declare no competing interests.

\section{ADDITIONAL INFORMATION}

Correspondence and requests for materials should be addressed to Stephanie D. Davis.

Reprints and permission information is available at http://www.nature.com/ reprints

Publisher's note Springer Nature remains neutral with regard to jurisdictional claims in published maps and institutional affiliations 